\title{
Study on the Innovative Way of Implementing Chinese Traditional Sports in the Situation of Nationwide Fitness
}

\author{
Xiyan Li \\ Tibet University, Lhasa 850000, Tibet, China
}

Keywords: National fitness; Traditional sports; Innovative way

\begin{abstract}
Since 2008, China has put forward the slogan of the National Fitness initiated by Olympics after successfully hosting the Olympics, which has opened a new charter for the Chinese traditional sports development and meanwhile, brought a new chance for nationwide fitness. Therefore, how to vigorously promote the development of Chinese traditional sports to encourage people participate in national fitness is a major research topic confronted by physical educator. So, this paper firstly analyzes the relation between characteristics of Chinese traditional sports and national fitness to further discuss its function. Finally, it comes up with an idea to promote the implementation of national fitness.

Throughout history, the physical and mental health of Chinese citizens is always considered as the important criteria and indicators to evaluate the development condition of a nation. Therefore, the suggestion of nationwide fitness can not only promote China's national sports development, but also promote the prosperity of our country, which makes a difference throughout the world. So, in the course of advocating nationwide fitness, new projects are also being initiated, hoping to find out a suitable national fitness and sports project such as running and balls in order to continuously popularize the overall sports abilities of the whole nation. However, if it is limited to the current situation, it is not enough and China has to continually search a suitable sports fitness project which is adapted to the current condition with promotion. Only in this way, can we promote the physical and mental fitness of Chinese citizens to develop and strengthen the national sports culture.
\end{abstract}

\section{Relation between Characteristics of National Traditional Sports and Nationwide Fitness}

Diversity. China is a multinational country with vast lands and colorful national sports. According to relevant statistics, there are more than 1000 traditional sports with different styles and characteristics. In addition, these sports are not only national but also have regional characteristics which can show the diversity of China's traditional sports [1] On the one hand, national sport is not only related to the local economy and culture but also has stable contents, location and time. For example, there is a windmill stepping activity on Lantern Festival hold by Maonan people or the archery contest on the second day of Lunar New Year hold by Li; on the other hand, national sport is also closely related to the traditional customs. For example, there are festivals ceremonies and celebrations in traditional sports or some traditional sports are integrated into the regional customs. Furthermore, national sports are mainly focused on entertainment which can not only create happiness but also encourage people to positively participate in the nationwide fitness to further promote people's life.

Body Building. Chinese traditional sports have a rich history and possess good qualities like self-cultivation, body building etc. For example, traditional martial arts pay attention to integration of motion and quietness, essence, energy, spirit in order to develop physical and mental health. What's more, Taijiquan highlights softness which has wide public base and is popular, adapted to the requirement of nationwide fitness. Especially in modern society, people suffer more in work, study and life, and sky rocketing people will have physical disease and mental disease. Under such condition, how to better cultivate physical and mental health and body building have become important issues. Comparing to fitness entertainment of western countries, Chinese traditional sports cannot only satisfy the entertainment need but also meet the requirement of nationwide fitness [2] Based on this we can fully play the entertainment feature of traditional sports but also satisfy the need for nature asked by modern people. 


\section{Function of Traditional Sports in Nationwide Fitness}

Promote the Development of Sports Industry. The development of traditional sports cannot only promote the nationwide fitness but also promote the development of sports industry. With the continuous development of time, people prefer an easy and recreational life, and sports tourism combing fitness and entertainment is popular among the public. China is a diverse nation, with terrific sceneries and abundant cultural geography resources while sports resources are outstanding. In the context of combing natural resources and national sports, the combination between traditional sports and tourism has become an excellent selling point. For example, Hui nationality of Guangxi vigorously developed regional sports such as bamboo dance, Sheng dance etc., which does not only promote the fitness activities but also promote the local tourism industry. ${ }^{[3]}$ Moreover, the dragon boat contest in Miluo River hold by Yueyang, Hunan does not only promote the international economy and trade development but also make the local sports culture famous. Furthermore, the sports industry has strong relations, which can develop the sports industry and facilitate the development of catering and clothes. Therefore, properly adopting traditional culture is also conducive to the economic and cultural development of the region.

Promote the Development of Education. National traditional sports provided in ethnic universities have superior advantages. On one hand, schools leaders and teachers are more familiar with the cultural customs of the region, therefore, they are able to impart knowledge of national sports for students, and promote the development of physical education activities; on the other hand, the equipment and faculties of ethnic universities can better satisfy the sports need of students. In addition, the prospective students of ethnic schools are mostly native residents, who are familiar with the traditional sports and are more interested in the sports comparing to students out of state. They have certain knowledge and can reduce the teaching pressure. ${ }^{[4]}$ The National Fitness Program also clearly clarifies to cultivate the students in ethnic schools and combines the economic requirement to promote nationwide fitness. Therefore, the traditional sports education in ethnic schools should be carried out based on the development of nationwide fitness. Besides, the theories should be deepened while sports education should be strengthened at the same time of teaching them national culture and knowledge so as to help students to fully understand the meaning of eternal sports teaching.

\section{Innovative Way to Implement Traditional Sports in the Situation of Nationwide Fitness}

Focus on the Talents Cultivation of Sport College. The implementation of national traditional sports in China should not only be organized by relevant departments to carry out management, supervision and inspection, but also needs to be integrated into national sports teaching in colleges, and combined with the syllabus so as to coordinate with material conditions of sports. In this way, students can be aware of the idea of nationwide fitness and lay a good foundation for life-long body building. The concept of life-long building is a trend of thought and its emergence is closely related to the development of modern sports. Therefore, we have to let students to be full aware of the meaning of life-long sports.

Besides, the reason why sports colleges exist is to cultivate professional sports talents with high quality and can be adapted to the sports development. Therefore, we should also pay attention to their quality. China's traditional sports are national and competitive therefore, we should also make them have strong ability to endure pressure and have perseverance, which can be helpful to improve their innovative ability and adjust their mental emotion, master sports skill and be adapted to social environment. ${ }^{[5]}$ So, concerning the different sports resources in different regions, we have to set different physical training requirement and integrate traditional sports concept into the team to better promote the idea of nationwide fitness. To fully combine the characteristics of modern society to strengthen the topic research of nationwide fitness, continually improve and renew should be of 
significant importance to develop nationwide fitness.

Strengthen Sports Education and Guide the Team Building. To strengthen the sports work, we have to build a good sports instructor team to better develop and promote the national sports. Therefore, China can fully take advantage of the traditional resources and further carry out the nationwide fitness under the leadership of relevant sports department so as to cultivate an excellent sports guidance team with professional skills, knowledge and organize ability. At the same time, we have to carry out regular training and improve their theoretical skill to carry out training schedule to continually standardize the actions. In this way, we can not only consolidate their practical skills as well as theoretical knowledge. In addition, we can check the learners based on activities and communicate to let them know the importance of nationwide fitness to both mental and physical health and encourage them to participate in it. ${ }^{[6]}$ So, we are able to attract more people to participate in nationwide fitness, and at the same time lay a good mass basis to the continuous development of traditional sports.

\section{Conclusion}

Traditional sports play a very important role in nationwide fitness. Traditional sports do not only have abundant natural resources and sports resources, the diverse regional features can also satisfy the fitness needs of the public. The nationwide fitness is closely related to traditional sports, which cannot be separated. In addition, the development of traditional sports is also the basis to realize nationwide fitness and can be considered as its ultimate goal while nationwide fitness also provides theoretical base for the development of traditional sports. Therefore, the government should lead the development of traditional sports in nationwide fitness to continually explore the culture of traditional sports so as to lead the implementation of nationwide fitness and provide larger development space to finally find out a way to develop traditional sports.

\section{References}

[1] X. Jiang, Innovative way to implement traditional sports in nationwide fitness, Journal of Wuhan Institute of Physical Education, 45 (2011) 51-54

[2] J. X. Wei, Y. R. Wang, Discuss on the innovative idea construction on combining nationwide fitness and traditional sports, Contemporary Sports Technology, 27 (2014) 154

[3] S. J. Lai, Discuss on the inheritance and innovation of minorities' traditional sports , Shaanxi Education: Higher Education, 7 (2011) 157

[4] Y. P. Meng, Probe into the function and promotion strategies to develop traditional sports in nationwide fitness, Sports, 9 (2012) 15-16.

[5] Z. G. Zhou, Analysis on the function of traditional sports in nationwide fitness, Education and Teaching Formula, 27 (2012)59-60.

[6] F. He, Important function of traditional sports in nationwide fitness, Science \& Technology of Stationery \& Sporting Goods, 10 (2014) 42 